\title{
The effectiveness of yttrium90/lutetium177-labeled somatostatin analogues treatment in functional pNETs
}

\author{
B. Michalik, D. Handkiewicz-Junak, K. Hasse-Lazar, B.Jarząb \\ Department of Nuclear Medicine and Endocrine Oncology. \\ Maria Skłodowska-Curie Memorial Cancer Center and Institute of Oncology, Gliwice Branch, Gliwice, POLAND.
}

\section{INTRODUCTION}

Therapy with somatostatin analoges is usually ineffective in attempt to remove hormonal symptoms in patients with functional pancreatic neuroendocrine tumors (pNET). Alternative treatment modalities, to control symptoms of excessive hormonal production, are necessary. Thus, the aim of our study was to evaluate results of radiopeptide treatment in patient with functional pNET.

\section{MATERIALS AND METHODS}

92 patients with pNET (49 women and 43 men, median age 58) have been observed for the last 10 years (2004-2014). Functional pNET were diagnosed in $21(23 \%)$ of them, including $9(10 \%)$ insulinomas, $4(4,5 \%)$ gastrinomas, 4 $(4,5 \%)$ glucagonomas, 2 (2\%) VIP-omas and $2(2 \%)$ with mixed hormonal activity.

\section{Distribution of neuroendocrine tumors of} the pancreas
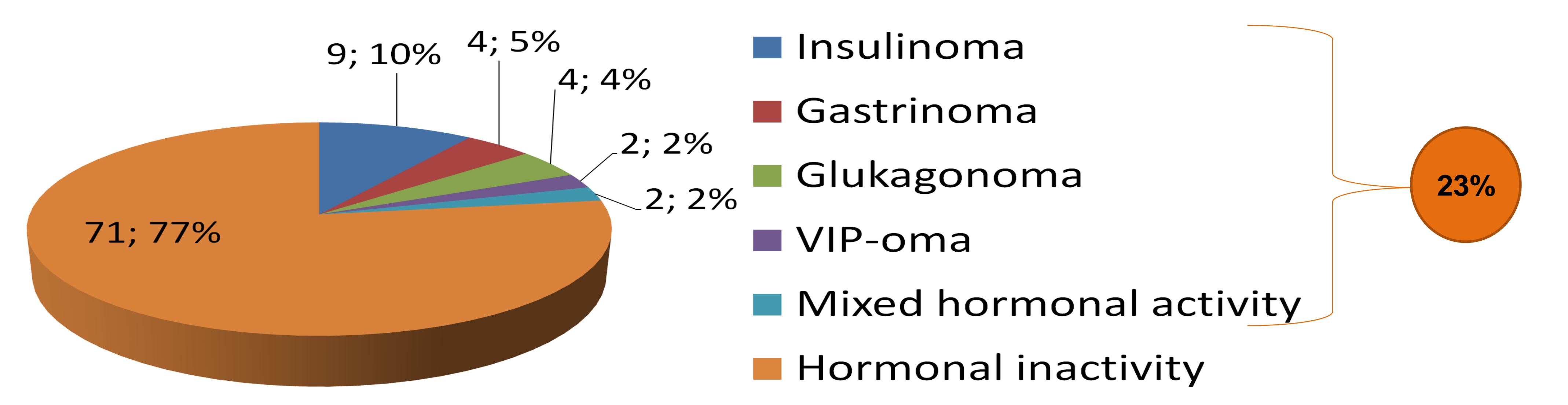

Seven patients with inoperable functional pNET and high expression of somatostatin receptor (confirmed in scintigraphy) were treated with radiolabelled (yttrium90/lutetium177) somatostatin analouges. At the time of PRRT start all patients were symptomatic since other treatment modalities were ineffective to control clinical symptoms of hormonal production.

Distribution of functional pNET in treated and untreated radiopeptide - Mixted hormonal activity $\quad$ VIP-oma Glucagonoma $\quad$ Gastrinoma $\quad$ Insulinoma

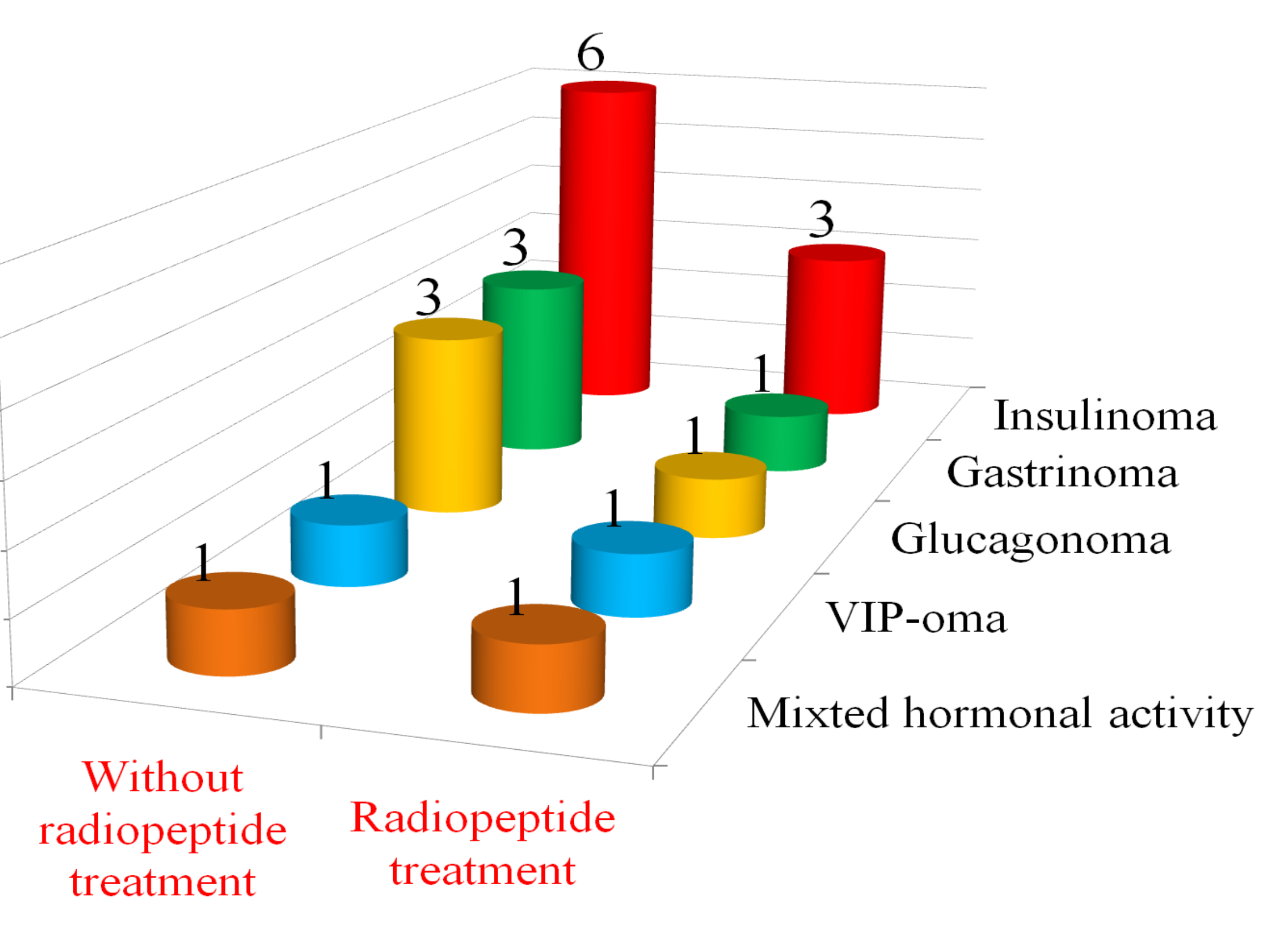

\section{RESULTS}

Clinical symptoms, resulting from excessive hormonal production, decreased after the first course of radiopeptide therapy and completely resolved at the end of the treatment in all treated patients.

Seven patients with functional pNET were treated with radiolabelled somatostatin analogues. Complete remission of hormonal activity ranged from 11 to 79 months (a median time of 36 months). Three subjects suffered from symptoms recurrence after a median time of 22 months from the ends of the therapy.

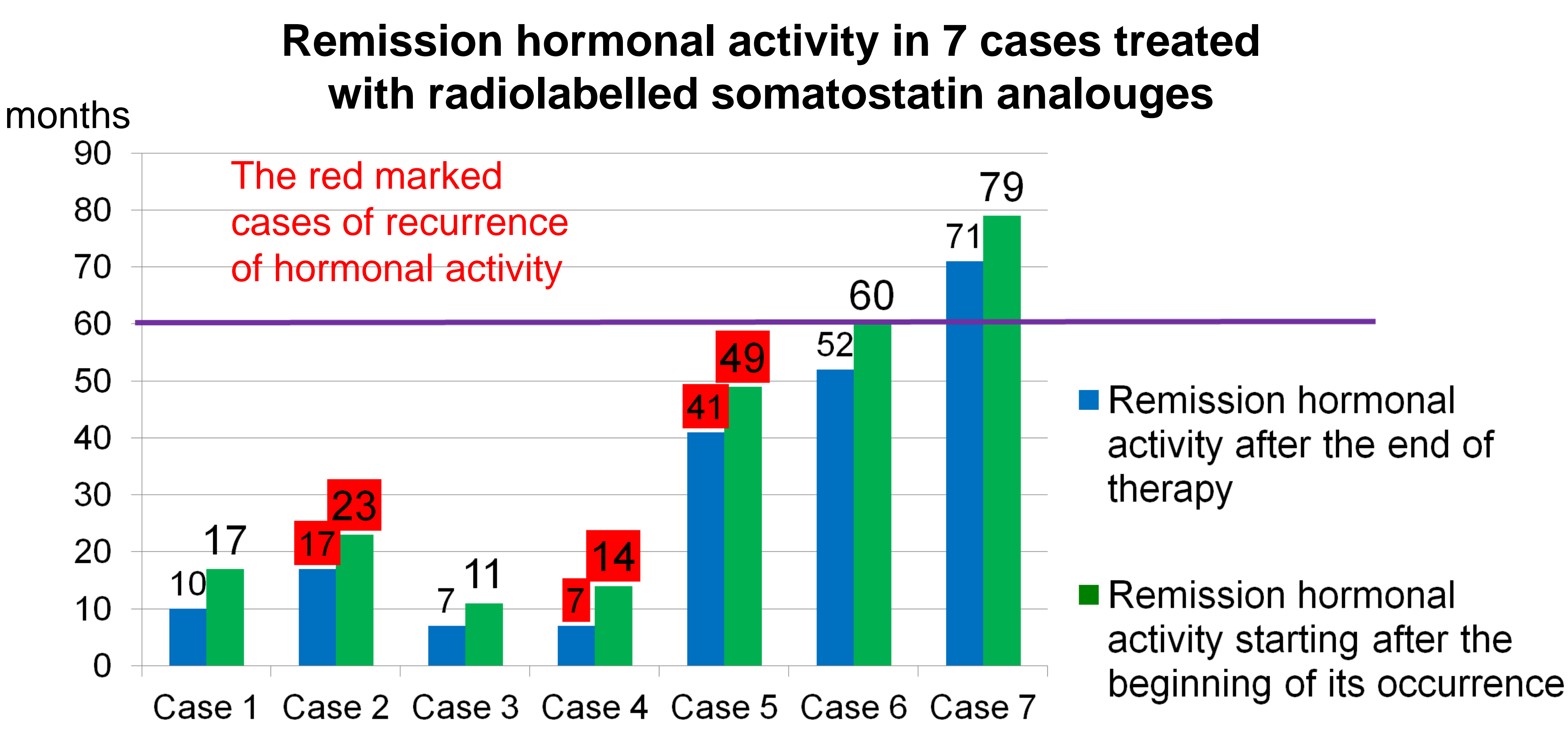

\section{Case studies}

1. Insulinoma. After PRRT symptoms of hypoglycemia resolved and the blood glucose normalized. Since the only symptom of the disease were liver metastases, liver transplantation was considered as an option for radical treatment.

2. Mixed hormonal activity (glukagonoma/insulinoma). PRRT allowed for regression of the symptoms of hypoglycemia with normalization of blood glucose. After 23 months the patient suffered from recurrence of hypoglycemia. Another 2 cycles of PRRT were ineffective in symptoms control and everolimus was started.

3. Insulinoma. Symptoms of hypoglycemia resolved with normalization of blood glucose levels.

4. Insulinoma. A patient remained without symptoms of hypoglycemia with normalization of blood glucose for $\mathbf{1 4}$ months after PRRT. Thereafter there was radiological progression and recurrence of symptoms.

5. VIP-oma. A patient with chronic diarrhea, dehydration and hypokalemia (due to the hormona activity of VIP-secreting tumors) with complete remission of symptoms after PRRT. Symptoms recurred 41 months after PRRT complition. The patients suffered from chronic kidney disease died of electrolyte disturbances.

6. Gastrinoma. Complete relief of gastritis (excessive secretion of gastrin) and patrial tumour regression acording to RECIST criteria was achieved.

7. Glucagonoma. After PRRT complete symptomatic and partial tumor regression (according to RECIST criteria) was achieved. After 6.5 years from the end of treatment there was a progression of metastatic retroperitoneal lymph nodes without symptomatic recurrence.

Clinical response was accompanied by tumor regression according to RECIST criteria in 3 patients (case 5,6,7).

\section{CONCLUSIONS}

Radiolabelled somatostatin analogues, administered in patients with functional pNET, allow for good control of clinical symptoms and objective tumor response. 